\title{
A NUMERICAL STUDY OF RELATIVISTIC JETS
}

\author{
J.A. FONT, J.M. MARTI AND J.M. IBÁÑEZ \\ Departamento de Astronomía y Astrofísica \\ Universidad de Valencia, 46100 Burjassot (Valencia), Spain
}

\author{
AND \\ E. MÜLLER \\ Max-Planck-Institut für Astrophysik \\ Karl-Schwarzschild-Str.1, 85740 Garching, Germany
}

Numerical simulations of supersonic jets are able to explain the structures observed in many VLA images of radio sources. The improvements achieved in classical simulations (see Hardee, these proceedings) are in contrast with the almost complete lack of relativistic simulations the reason being that numerical difficulties arise from the highly relativistic flows typical of extragalactic jets. For our study, we have developed a two-dimensional code which is based on (i) an explicit conservative differencing of the special relativistic hydrodynamics ( $\mathrm{SRH}$ ) equations and (ii) the use of an approximate Riemann solver (see Martí et al. 1995a,b and references therein).

The SRH equations have been solved in normalized form by setting the light speed $c$, the beam radius $R_{\mathrm{b}}$, and the rest-mass density of the external medium $\rho_{\mathrm{em}}$ equal to unity. The flow is then completely characterized by five dimensionless parameters: the beam to external medium rest-mass density ratio, $\eta \equiv \frac{\rho_{b}}{\rho_{e m}}$, the pressure ratio, $K \equiv \frac{p_{b}}{p_{e m}}$, the beam velocity, $v_{\mathrm{b}}$, the beam Mach number, $M_{\mathrm{b}} \equiv \frac{v_{b}}{c_{s}}$ and the adiabatic exponent, $\gamma$. In our study these parameters take the following values: $\eta=0.01,0.1,1, K=1$, $v_{b}=0.9,0.99,0.999, M_{b}=M^{\text {min }}, 6.0$ and $\gamma=4 / 3,5 / 3$. Models with $M_{b}$ near the minimum value, $M^{\text {min }} \equiv v_{b} / \sqrt{\gamma-1}$, have large internal energies compared with their rest-mass energy. They are called hot models, while highly supersonic jets are referred to as cold models.

In all our simulations the jets show the gross morphological features already found in non-relativistic calculations (see, e.g., Norman et al. 1982). We find important differences between hot and cold relativistic jets. In hot models the Mach shock at the jet head is permanently present during the 
whole simulation. They show naked beams surrounded by lobes instead of cocoons. In hot jets the backflow appearing at the working surface is minimized or, as $v_{b}$ increases, even non-existing. In addition, they almost completely lack internal structure, because of the pressure equilibrium between the beam and its surroundings. Therefore, the beam/cocoon interface of hot jets is very stable against the growth of pinch instabilities that would evolve into internal shocks. In cold models the Mach configuration is temporarily substituted by a cross-shock during the evolution. These shocks are not as efficient as normal shocks in decelerating the beam flow allowing it to push the contact discontinuity and, eventually, the bow shock. This effect is more important for beams with a lower value of the adiabatic exponent. Backflow is more important in cold models, where stable cocoons can be found in the early stages of the evolution. These cocoons eventually evolve into vortices producing turbulent structures. In cold jets with $\gamma=5 / 3$ the cocoon is mainly formed by large vortices while in models with $\gamma=4 / 3$ the strong beam collimation causes a large acceleration of the jet. Thus, beam gas is less efficiently redirected into the cocoon, and thinner cocoons with smaller vortices form. Cold models present also a complex structure of internal shocks generated by pressure mismatches between the beam and the overpressured cocoon and by perturbations of the beam boundary by vortices and bulk motions within the cocoon.

For sufficiently large beam flow speeds the estimated propagation velocity of the jet head, obtained by equating the ram pressure of the beam and the external medium in the proper frame of the working surface (see Martí et al. 1994), approaches that of the beam itself. In classical dynamics this only occurs for so-called ballistic jets (i.e. $\eta \gg 1$ ). Similarly as for classical jets, we define the propagation efficiency $\delta$ as the ratio between the mean jet velocity and its corresponding estimate. Our results show (Martí et al. 1995b) that for a wide range of estimated jet propagation speeds $(0.17 c-0.94 c)$ the efficiencies span the interval $0.76-1.24$, i.e. they are significantly larger than their corresponding Newtonian counterparts. We find that hot models have $\delta$ very close to one. Highly supersonic (i.e. cold) models with $\gamma=4 / 3$ have $\delta$ greater than one due to the acceleration phase while in models with $\gamma=5 / 3$ the efficiency increases with $v_{b}$ and $\eta$, and tends to one for sufficiently dense, highly relativistic models.

\section{References}

Hardee, P (1995) IAU Symposium 175: Extragalactic Radio Sources Martí, J. Maㅡ, Müller, E., Ibáñez, J. Ma (1994) $A \mathcal{E} A, 281$, L9

Martí, J. M므, Müller, Font, J.A., Ibáñez, J. Mํㅡ (1995) $A p J, 448$, L105

Martí, J. Mํㅡ, Müller, Font, J.A., Ibáñez, J. Maㅡ, Marquina, A. (1995) $A p J$ (submitted)

Norman, M. L., Smarr, L., Winkler, K.-H. A., Smith, M. D. (1982) $A \& A, 113,285$ 